\title{
Obesity: a chronic disease
}

\section{Statistics $20 \mid 7$}

Worldwide, obesity has almost tripled since 1975. In 2017, more than 2 billion adults, were overweight. Of these 650 million were obese $39 \%$ of adults aged 18 years and over were overweight in 2016 and $13 \%$ were obese. Most of the world's population lives in countries where overweight and obesity kill more people than malnourished. 41 million children under the age of 5 were overweight or obese in 2016. More than 340 million children and adolescents aged 5-19 were overweight or obese in 2016.

\section{Etiopathogenesis}

As with any other disease, the causes of obesity are a combination of genes, endocrine mechanisms, environmental factors such as stress, nutrition, increasing sedentary life and the obesogenic environment. More than 30 genes in 12 chromosomes appear to be related to the Body Mass Index.

\section{Work-way of life-urbanization}

The average European has about 5 hours of sedentary work / day. The amount of calories that an employee spends a day in his work has been reduced (since 1960) by $15 \%$ due to sedentary work. $60 \%$ of Europeans use the car for their daily transport. It is estimated that in $205080 \%$ of Europeans will live in urban areas. Larger portions: the portion in soft drinks with sugar and snacks has increased up to $+100 \%$ (1960). We consume an average of 500kcal / day since 1960 . $42 \%$ of Europeans do no exercise.

\section{Symptoms}

Often overweight and mostly obese, we treat have clinical
Volume 9 Issue 2 - 2019

\section{Katerina A Chremou \\ Clinical Dietitian, Nutritionist, Greece}

Correspondence: Katerina A Chremou, MSc, Clinical Dietitian -Nutritionist, Greece, Email k.chremou@hotmail.com

Received: February 28, 2019 | Published: April 02, 2019

symptoms from other systems with the symptoms of obesity. There are also healthy obese adults without disturbed biological indices (hypertension, diabetes, diabetes etc.). According to the World Health Organization (WHO) the symptoms of obesity are: Increased appetite, Lack of feelings of food saturation, Shortness of breath, Increased sweating, Rattling, Unable to deal with sudden physical activity, Feeling of daily fatigue, Back and joint pains, Low self-esteem and self-esteem, A feeling of isolation from social groups.

\section{Clinical events}

According to the World Health Organization, therefore, the increase in body weight and consequently in the Body Mass Index (BMI) is directly related to the possible development of chronic illnesses and the reduction of life expectancy. In the figure above, the main effects of obesity are outlined. It should be noted that the mechanisms of development of the various markers are linked to one another with the main one of the chronic inflammation (Figure 1). ${ }^{1-3}$

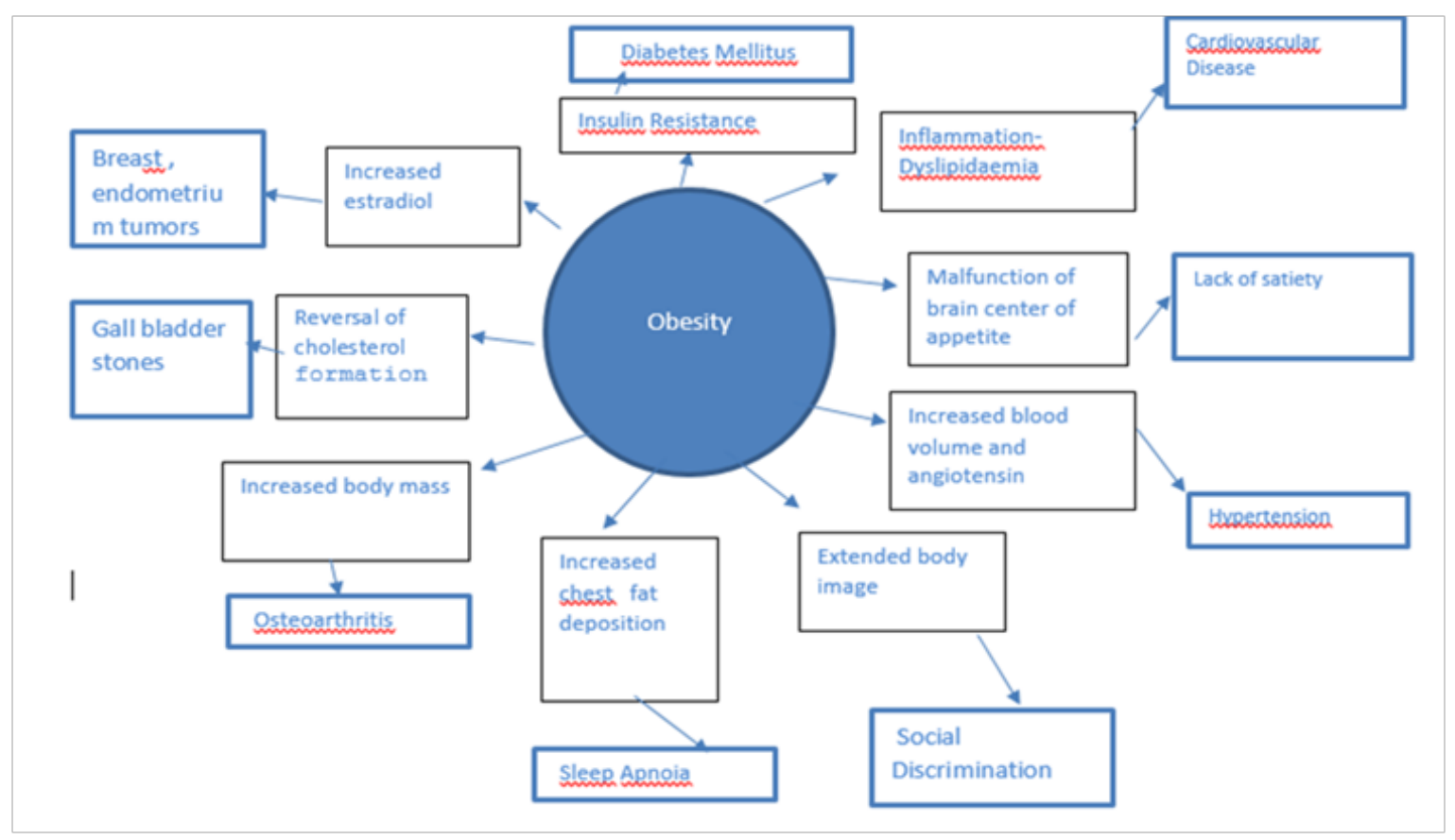

Figure I Obesity. 
From the above, we conclude that obesity should be treated not only with Body Mass Index (BMI), but as a chronic recurrent disease, such as diabetes or hypertension, characterized by abnormally distributed or excess body fat that affects health. The theory that obesity is a chronic disease is not only related to the somatometric indicators but also to other health indicators (biomedical, metabolic, spiritual and social). As a result of social stigma, surveys have pointed out that obese adults have an impact on their body, and this leads them to: Low self-esteem, increased rates of depression, and in some cases suicidal tendencies, increased incidence of eating disorders (Bulimia, night overeating), reduced physical activity, avoiding and refusing medical assistance,exclusion-Isolation.

\section{Conclusion}

As a result of endocrine and neuroendocrine regulation, the body resists the loss of body weight. This is one of the reasons that obesity is considered a chronic illness. It requires lifelong management, like other chronic diseases. and the application of both medical, dietetic and behavioral techniques for timely treatment.

\section{Acknowledgments}

None.

\section{Conflicts of interest}

The authors declared there is no conflict of interest.

\section{References}

1. Bray GA, Kim KK, Wilding JPH. World Obesity Federation Position Statement: Obesity: a chronic relapsing progressive disease process. A position statement of the World Obesity Federation. Obes Rev. 2017;18:715-723.

2. European Obesity day. EASO (European Association for the study of Obesity). 2016.

3. Weight stigma in health care. 